\title{
A novel customized ceramic bracket for esthetic orthodontics: in vitro study
}

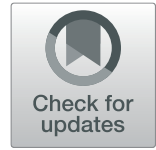

Liu Yang ${ }^{1}$, Guangfu Yin ${ }^{1^{*}}$, Xiaoming Liao ${ }^{1}$, Xing Yin ${ }^{2^{*}}$ and Niansong Ye ${ }^{3}$

\begin{abstract}
Background: This study aims to develop a novel process to establish a standardized manufacturing technique of customized esthetic ceramic bracket system (CCB) which could be endowed with individual color and shape to satisfy patients' individual demands. Material characteristics and mechanical parameters of CCB were evaluated.

Subjects and methods: CCB virtual models were designed individually according to patient's teeth morphology and clinical demands. 3D printing technology, lost-wax technology, and selected glass-ceramic ingots were employed to fabricate CCB. Scanning electron microscopy (SEM) analyses were performed to characterize the surface morphology of CCB and commercially available brackets (Clarity Advanced; Crystalline VII; Inspire ICE; Damon Q). Static and kinetic frictional resistance (FR), shear bond strength (SBS) and adhesive remnant index (ARI) scores were recorded. One-way analyses of variance (ANOVA) and post-hoc Tukey's HSD multiple tests were used for statistical analyses.

Results: Multi-color and multi-transparency raw materials facilitated CCB with a wide range of color options and controllable optical properties to satisfy different esthetic demands of individual orthodontic patients. CCB presented same level of FR as commercially available ceramic brackets did. No significant differences $(P \geq 0.05)$ of SBS were observed among CCB-ES (treated silane), Clarity Advanced and Crystalline VII groups, and CCB-E (no silane) attained the highest ARI mean score 3. In the preliminary clinical trial, CCB presented excellent colormatching and shape-matching appearances similar to natural teeth, which made it highly invisible from social intercourse distance.
\end{abstract}

Conclusions: CCB were demonstrated to be an applicable labial orthodontic bracket system with optimized esthetics and biomechanics. We envision that it would be an ideal alternative for patients who pursue esthetic orthodontic treatment but were not likely to take lingual appliances or clear aligners.

Keywords: Customized ceramic brackets, Esthetic orthodontics, Lithium disilicate, 3D printing, Heat-pressing

\section{Introduction}

As it happens in contemporary dentistry, orthodontics is undergoing significant development attributed to digital technologies, which influences the diagnosis, planning and the entire process of orthodontic treatment $[1,2]$. A novel customized system allows for assessment of changes in 3D and customization of treatment planning brackets, and wires by means of intraoral scanning,

\footnotetext{
* Correspondence: nic0700@scu.edu.cn; yinxing@scu.edu.cn

${ }^{1}$ College of materials science and engineering, Sichuan University, No.24 South Section 1, Yihuan Road, Chengdu, China

${ }^{2}$ State Key Laboratory of Oral Diseases, National Clinical Research Centre for Oral Diseases, West China Hospital of Stomatology, Sichuan University,

Chengdu, No. 14, South Renmin Road, Chengdu, China

Full list of author information is available at the end of the article
}

cone-beam computed tomography (CBCT), threedimensional (3D) photography, and computer-aided design and computer-aided manufacturing (CAD/CAM) technologies [3, 4]. At present, a wide variety of customized orthodontic appliances, including metal labial or lingual brackets and clear aligners are developing rapidly and receiving growing attention. Meanwhile, customized appliances have caused tremendous threat to traditional orthodontic brackets [5, 6]. Fixed customized appliances have demonstrated favorable treatment outcome, enhanced patient comfort and significantly reduced chair time and total treatment time [7-10]. Nevertheless, metal labial fixed appliances increase the visual perception on patients' teeth by the distinct appearance, and 
there are reports that lingual brackets still induce discomfort in a certain proportion of patients [11, 12].

Ceramic bracket was introduced in 1980 s as a more esthetically pleasing alternative to the metallic brackets [13]. Currently available ceramic brackets are almost composed of aluminum oxides and reveal high strength, chemical stability and biocompatibility [14]. With the improvement of ceramic bracket performances, the demand for esthetics orthodontic became the main target [15]. Less visual perception, to which the term esthetic is related, was desired. And the esthetic performances of ceramic brackets are influenced by optical properties of patients' teeth. To achieve an esthetic performance, brackets should match the tooth color and/or possess proper translucency to allow the underlying tooth color to penetrate through them, making the brackets less noticeable [16, 17]. Technically and economically, it is much harder to fabricate personalized (shape and appearance) ceramic appliances than metallic appliances with conventional forming approaches, whether polycrystalline or monocrystalline ceramic brackets. To our knowledge, no customized ceramic appliances manufacturing technique has been well-established, even though various brands of commercially available ceramic brackets, e.g. Clarity Advanced, Inspire ICE, Crystalline VII, InVu, have been employed for decades.

Additive manufacturing refers to $3 \mathrm{D}$ printing technique that enables the manufacturing of a wide range of products with specific properties and shapes that meet patients' demands [18]. Although 3D printing has achieved some clinical applications in orthodontics [19], surface quality, dimensional accuracy and the mechanical properties still need to be improved in order to allow fabricating customized ceramic orthodontic appliances.

Customization and esthetics are considered as critical factors both in dental restoration and orthodontic filed. All-ceramic materials are well known as ideal dental restoration materials due to the esthetic ability of imitating tooth color. As a representative glass-ceramic material, lithium disilicate material IPS e.max system (Ivoclar Vivadent AG, Schaan, Liechtenstein) reveals a higher fracture toughness and flexural strength [20], and allows dental technicians to customize dental restorations in terms of form and esthetics by heat-pressing or machining technology. Heat-pressing technology allows a more complex shape and less cost for custom fabrication, such as crowns, veneers or bridges, etc. To date, the application of lithium disilicate in the orthodontic filed, however, has not been reported before.

Thus, the aim of this study is to develop a novel process to establish a standardized manufacturing technique of customized esthetic ceramic orthodontic bracket system (CCB), which employed the individual digital design, lithium disilicate materials and heatpressing technology. The morphology, friction resistance (FR) and shear bond strength (SBS) of CCB were evaluated in comparison with commercially available ceramic bracket products.

\section{Materials and methods \\ The design and manufacture of CCB}

Figure 1 shows that the manufacture processes of $\mathrm{CCB}$ virtual models involves as follows. Digital models of patient dental arches were obtained via an intraoral scanner (TRIOS 3 Basic, 3 Shape, Denmark) and CBCT (KaVo 3D eXam-i, Kava Sybron, Germany) (Fig. 1a). After virtual teeth arrangement (Fig. 1b), customized brackets which match the morphology of teeth were generated on archwire plane (Fig. 1c). Finally, a sprue was added to each of the bracket model on the side face (Fig. 1d) and the virtual model was exported as a standard triangulation language (STL) file.

High-resolution rapid digital light processing (DLP) technology printer was used to convert the virtual bracket models into wax patterns (Fig. 2a). Glass ceramic ingot (IPS e.max press, Ivoclar Vivadent AG, Liechtenstein), matching the color of patient teeth, was chosen by using a shade guide. Sprueing, investing, preheating, and pressing procedures (Fig. 2b) were carried out according to the manufacturer's recommendations of dental restorations. Then brackets were divested (Fig. 2c), polished, characterized, glazed, and sintered (Fig. 2d). Finally, ruby oilstone slices with 0.22-in thickness were used to polish the slot of bracket, till a smooth slot looking was achieved.

\section{Specimen preparation}

As shown in Table 1, besides CCB, polycrystalline alumina ceramic brackets Clarity ADVANCED (CA) and Crystalline VII (C7), monocrystalline alumina ceramic bracket Inspire ICE (IC) and a self-ligating metal bracket Damon Q (DQ) were used for in vitro study. All bracket samples were fabricated for maxillary first premolar and contained a 0.022 -in slot. Two dimensions of rectangular $0.018 \times 0.025$-in and round 0.016-in straight stainless steel archwires were chose for FR testing.

\section{SEM evaluation}

Brackets and archwires morphologies were analyzed by field emission scanning electron microscopy (S-4800, Hitachi, Tokyo, Japan). Low magnification SEM images illustrate the general structure and high magnification SEM images show the microcosmic surface topography.

\section{Frictional resistance tests}

To compare the FR level of CCB with other ceramic brackets, 80 brackets (20 CCB, 20 CA, 20 C7, 20 IC) and 80 SS archwires $(40$ rectangular $0.018 \times 0.025$-in 
a

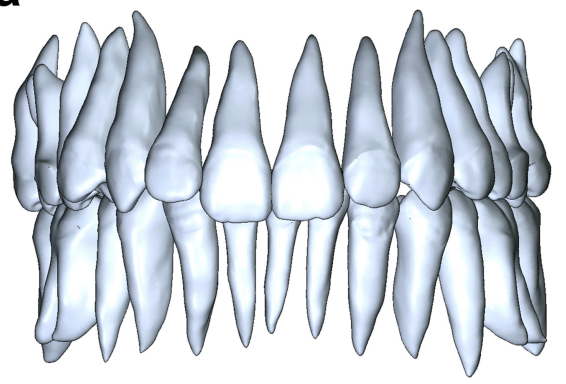

C

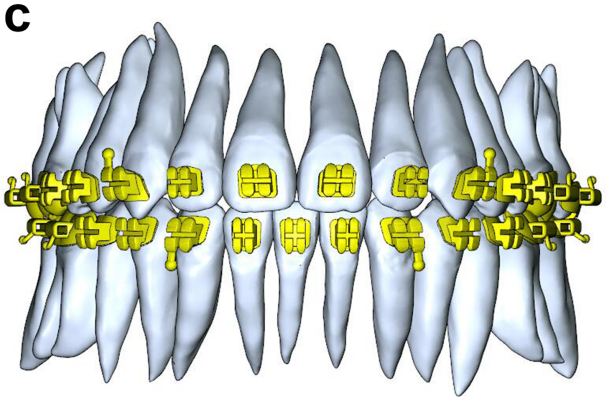

b

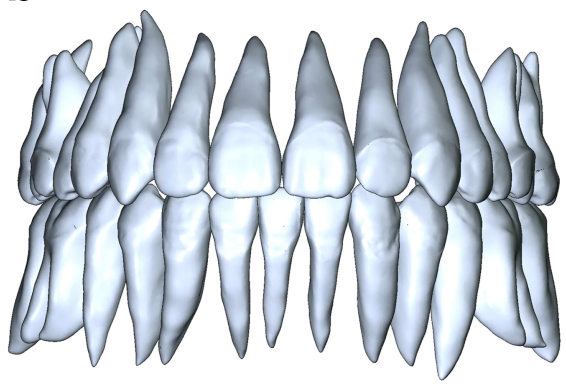

d

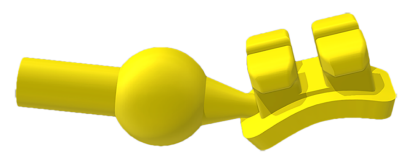

Fig. 1 Virtual construction of customized bracket modeling: a digital modeling of initial teeth; $\mathbf{b}$ digital modeling of rearrangement teeth; c generating customized bracket bases and body; $\mathbf{d}$ separating bracket and adding a sprue on side face

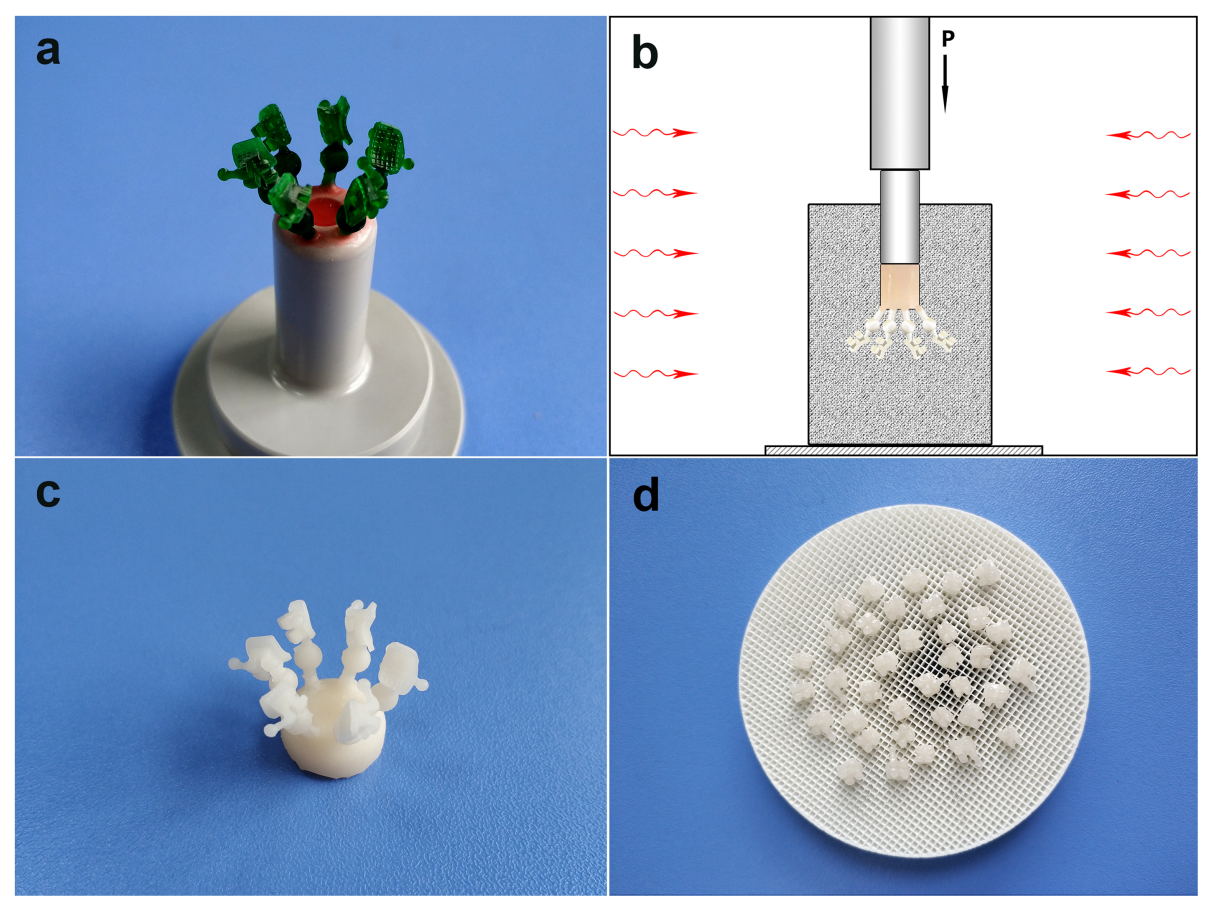

Fig. 2 Manufacture processes: a wax patterns of customized ceramic bracket; $\mathbf{b}$ principle diagram of heat-pressing procedure; $\mathbf{c}$ divesting after heat-pressing; $\mathbf{d}$ sintering after glazing 
Table 1 Materials evaluation

\begin{tabular}{lllllll}
\hline Trade name & Materials & Abbr & Slot / wire dimension & Torque & Angulation & Manufacture \\
\hline- & Lithium disilicate & CCB & 0.022 -in & $-7^{\circ}$ & $0^{\circ}$ & Self-made \\
Clarity ADVANCED & Polycrystalline alumina & CA & 0.022 -in & $-7^{\circ}$ & $0^{\circ}$ & 3 M/Unitek, CA, USA \\
Crystalline VII & Polycrystalline alumina & C7 & 0.022 -in & $-7^{\circ}$ & $0^{\circ}$ & Tomy Inc., Tokyo, JP \\
Inspire ICE & Monocrystalline alumina & IC & 0.022 -in & $-7^{\circ}$ & $0^{\circ}$ & Ormco Corporation, CA, USA \\
Damon Q & Stainless steel & DQ & 0.022 -in & $-11^{\circ}$ & $2^{\circ}$ & Ormco Corporation, CA, USA \\
Remanium & Stainless steel & 16 & 0.016 -in & - & - & DENTAURUM, Ispringen, DEU \\
Remanium & Stainless steel & $18 \times 25$ & $0.018 \times 0.025$-in & - & - & DENTAURUM, Ispringen, DEU \\
\hline
\end{tabular}

archwires and 40 round 0.016-in archwires) were used for this study. Besides, diameter of $0.2 \mathrm{~mm}$ stainless steel ligatures and general elastic ligatures were used for archwire ligation. Bracket-wire-ligation combinations were divided into 16 subgroups $(n=10)$ of four bracket types, two wire types and two ligatures. Each bracket was positioned and bonded on a glass slide. The slot was remained in a horizontal position with the long axis of glass slide. A universal testing machine (EZ-LX 1kN, Shimadzu, Kyoto, Japan) was used to investigate the FR in a dry state. Glass slide was fixed to the lower jig of universal testing machine in an upright direction. The angulation between the bracket slot and archwire was $0^{\circ}$, and the archwire was pulled through the slot for $5 \mathrm{~mm}$ distance at a crosshead speed of 5 $\mathrm{mm} / \mathrm{min}$. For each test of SS ligature (SSL) and elastic ligature (EL), a common bracket was evaluated against the fresh surface of a common wire. The same operator prepared the specimens and performed all tests of this study. Static and kinetic friction forces were measured. The static friction force was determined by the first force peak, while the kinetic friction force was calculated as the mean force from the peak until the end of test.

\section{Shear bond strength tests}

Sixty healthy human maxillary first premolars were prepared and stored at room temperature in $0.1 \%$ (wt/vol) thymol solution. Before bonding, roots of teeth were embedded in autopolymerizing acrylic resin with cylindrical mold. Base areas of all brackets were estimated by weighing uniform pieces of lead foil, which was applied to precisely cover the base surface of each bracket. Brackets were treated and divided into six groups $(n=$ 10) as following:

Group CCB-E: 5\% hydrofluoric (HF) acid gel (IPS Ceramic Etching Gel, Ivoclar Vivadent AG, Schaan, Liechtestein) was applied on the base of CCB for $20 \mathrm{~s}$, rinsed with water spray for $10 \mathrm{~s}$, dried with oil-free air.

Group CCB-ES: Contrasted with group CCB-E, after HF gel etching, all brackets base were subjected to silane coupling agent (Monobond N, Ivoclar Vivadent AG,
Schaan, Liechtestein) for $60 \mathrm{~s}$ and rinsed with oil-free air.

Group CA, C7, IC, DQ: No treatments were conducted on bracket base except for cleaning up in absolute ethyl alcohol.

The labial surfaces were etched with $37 \%$ phosphoricacid gel (Eco-Etch, Ivoclar Vivadent AG, Schaan, Liechtestein) for $30 \mathrm{~s}$, then rinsed with water spray for $10 \mathrm{~s}$, and dried lightly with oil-free compressed air finally. Adhesive primer (Transbond $^{\mathrm{Tm}} \mathrm{XT}, 3 \mathrm{M}$ Unitek, Monrovia, USA) was rubbed onto the enamel surface of all group teeth. Next, brackets were bonded on the teeth surface with a light-cure composite resin (Transbond ${ }^{\mathrm{TM}} \mathrm{XT}, 3 \mathrm{M}$ Unitek, Monrovia, USA), and care was taken to express the composite evenly on all sides. Superfluous adhesive was carefully removed from the periphery of the bracket base with an explorer. All specimens were light-cured with a curing light (Wave length: $420 \mathrm{~nm}-480 \mathrm{~nm}$ ) for $20 \mathrm{~s}$. Samples were stored in an isotonic saline solution at $37^{\circ} \mathrm{C}$ for $24 \mathrm{~h}$. After 1000 times $5-55^{\circ} \mathrm{C}$ thermacycling and 15-s dwell time, shear bond testing was conducted using a universal testing machine (AG-IC $20 \mathrm{kN}$, Shimadzu, Kyoto, Japan) at a crosshead speed of $5 \mathrm{~mm} /$ min. After SBS tests, the bond failure bracket bases were examined by SEM. The adhesive remnant index (ARI) score was used to determine the amount of adhesive remaining on the specimen surface [21].

\section{Statistical analysis}

One-way analysis of variance (ANOVA) and post-hoc Tukey's HSD multiple tests were used to test the significance of differences of FR and SBS values. The results were considered to be significant at $P$-values below 0.05 $(P<0.05)$.

\section{Results}

\section{Appearance and morphology}

As shown in Fig. 3a, CCB-a, CCB-b and CCB-c were manufactured with Impulse-O1, HT-A1 and HT-C4 ingots, respectively. With the change of glass-ceramic ingots, the colors of $\mathrm{CCB}$ gradually darkened to faint yellow color in natural light, transparency tended to be 


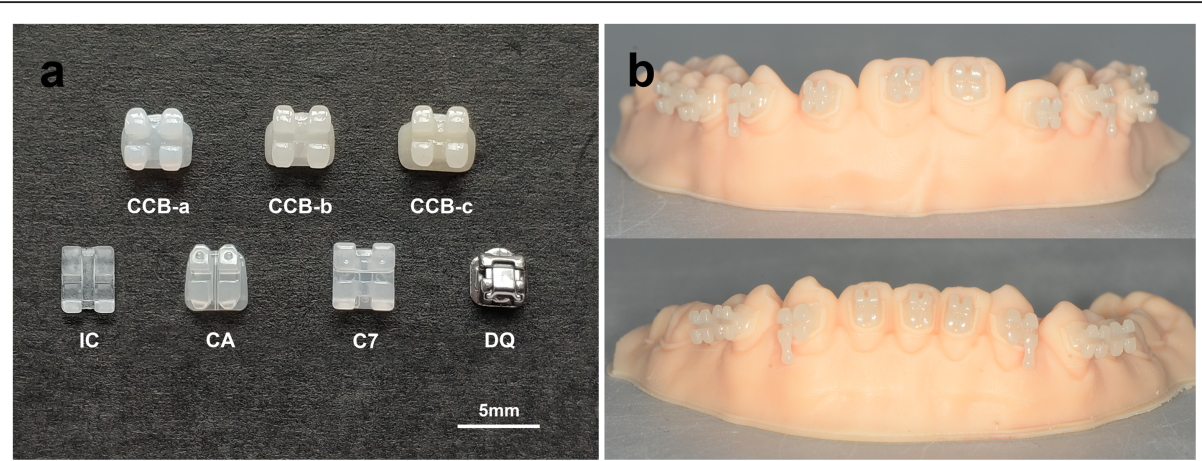

Fig. 3 Images of brackets: a the different opacity CCB (CCB-a, CCB-b, CCB-c) and commercial brackets (IC, CA, C7 and DQ); b customized ceramic brackets bonded on teeth model for fabricating indirect transfer jigs

opaque. The similar appearances were detected among CCB-a, CA and C7. Compared with the other brackets, CCB-b and CCB-c displayed a darker appearance similar to normal natural human teeth color. A set of maxillary and mandibular CCB were bonded on dental cast by experienced technician to ensure accuracy for fabricating indirect bonding transfer jigs (Fig. 3b).

Figure 4 shows the different morphologies of brackets and archwires. No obvious defects were found at bracket surfaces (Fig. 4a, e), and various base structures and morphologies were detected (Fig. 4f, j). CCB, CA, C7, IC and DQ base comprised grid pits, randomly oriented sharp crystals, grooves, mass spherical particles and mesh respectively. Smooth surfaces of archwires are observed in Fig. 4k, 1. It was obvious that different manufacturing processes and designs resulted in various surface morphologies of slot. After being rasped with ruby oilstone, smooth plane contained some parallel traces and small pits were detected on CCB slot surface (Fig. 5a). CA and C7 slots both revealed smooth and polycrystalline surface, but the grains of $\mathrm{CA}$ were smaller and more uniform in comparison with C7 (Fig. 5b, c). Mass constant-thickness step structures were observed on IC slot surface (Fig. 5d).

Figure 6 depicts representative morphology of debonding bases. Relatively clear surface was found on CCB-E

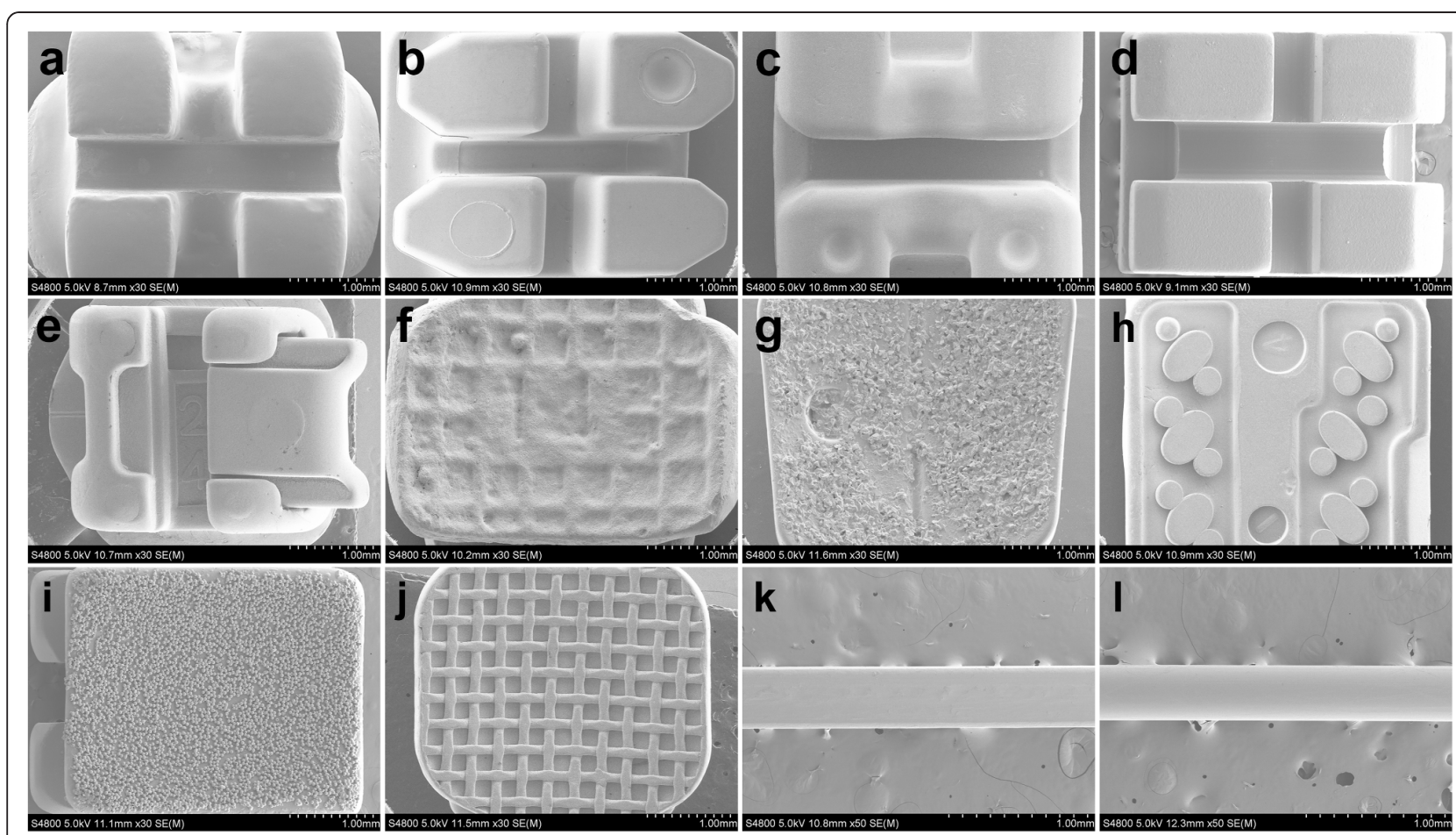

Fig. 4 SEM images of bracket bodies, at 30x: a CCB; b CA; c C7; d IC. e DQ. Bracket bases, at 30X: f CCB; $\mathbf{g} C A ; \mathbf{h} C 7 ; \mathbf{i} I C ; \mathbf{j}$ DQ. Stainless steel archwires, at 50x: $\mathbf{k}$ edgewise archwire; I round archwire 


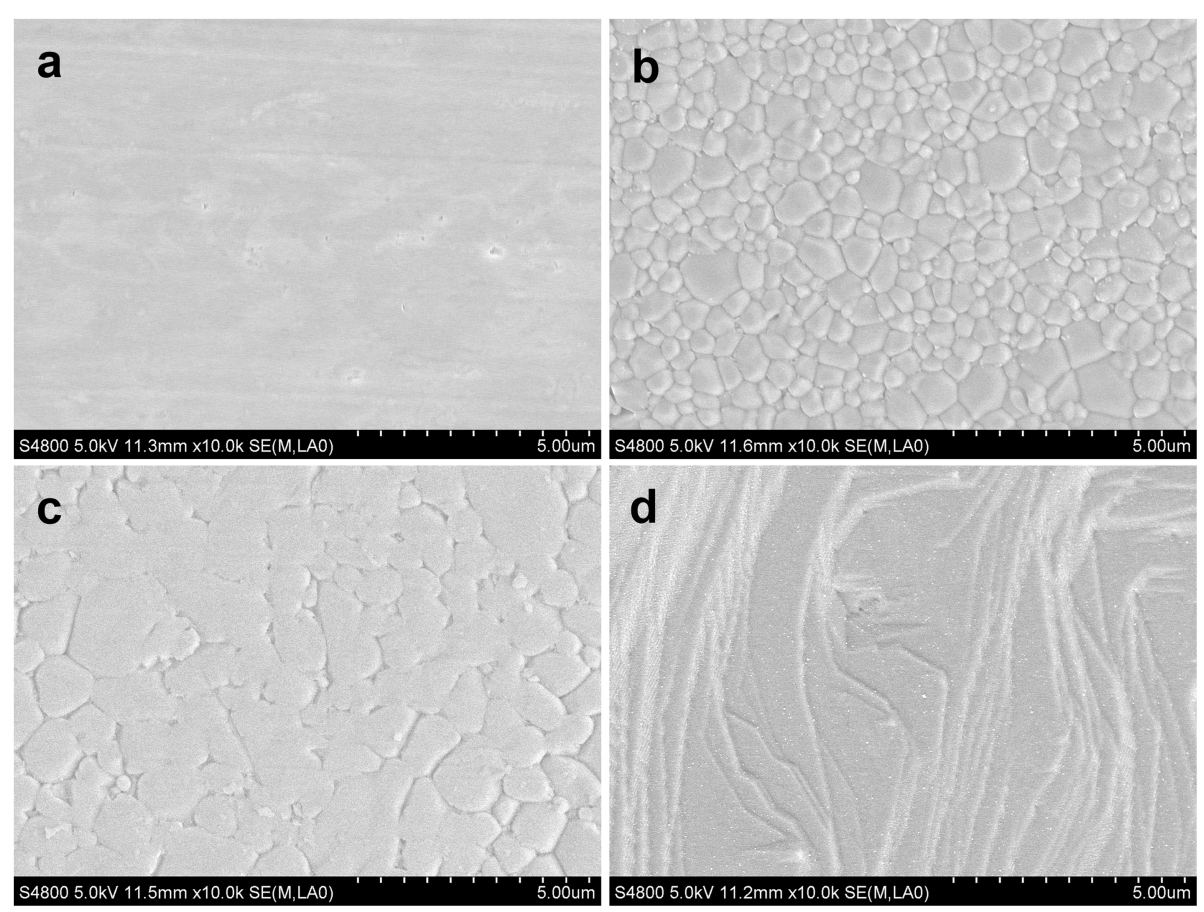

Fig. 5 SEM images of ceramic bracket slot, at $10 \mathrm{kx}$ : a CCB; b CA; c C7; d IC

base (Fig. 6a). In comparison, some large adhesive remnants were detected on CCB-ES (Fig. 6b), Crystalline VII (Fig. 6d), Inspire ICE base (Fig. 6e) and Damon Q base (Fig. 6f). Figure 7a-b display the difference of $\mathrm{CCB}$ base before and after HF etching. A rough topographic was detected on initial base (Fig. 7a). After etching, base surface showed a scaffold-like microstructure which comprises some small interlocking needle-like crystals without orientation (Fig. 7b). The break failure of CCB-E occurred at the resin-ceramic interface, and not any effective bonding at resin-ceramic interface was observed but some micro-retentions of adhesives in the irregular

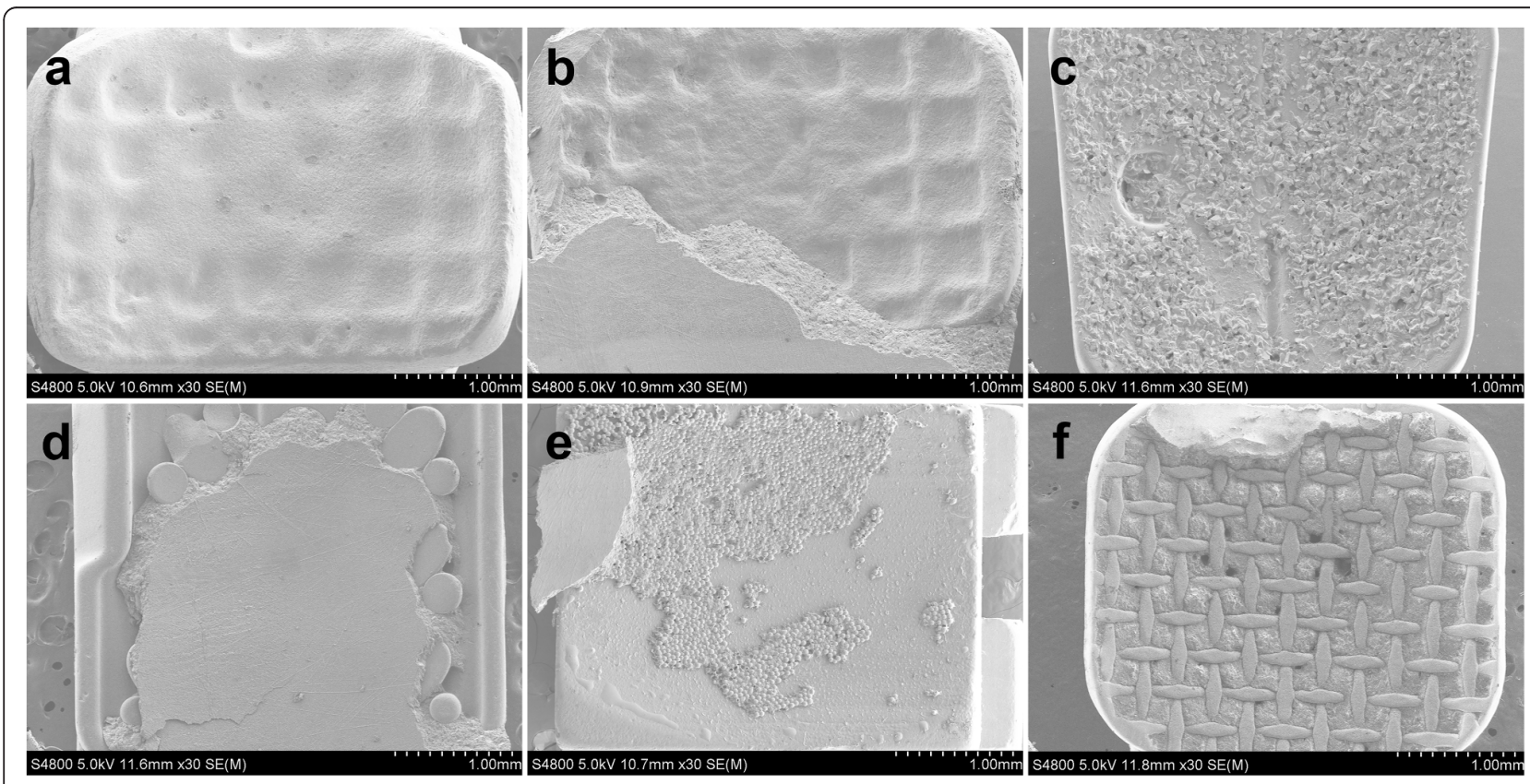

Fig. 6 SEM images of debonding bases at 30X: a CCB-E; b CCB-ES; c CA; d C7; e IC; $\mathbf{f} D Q$ 

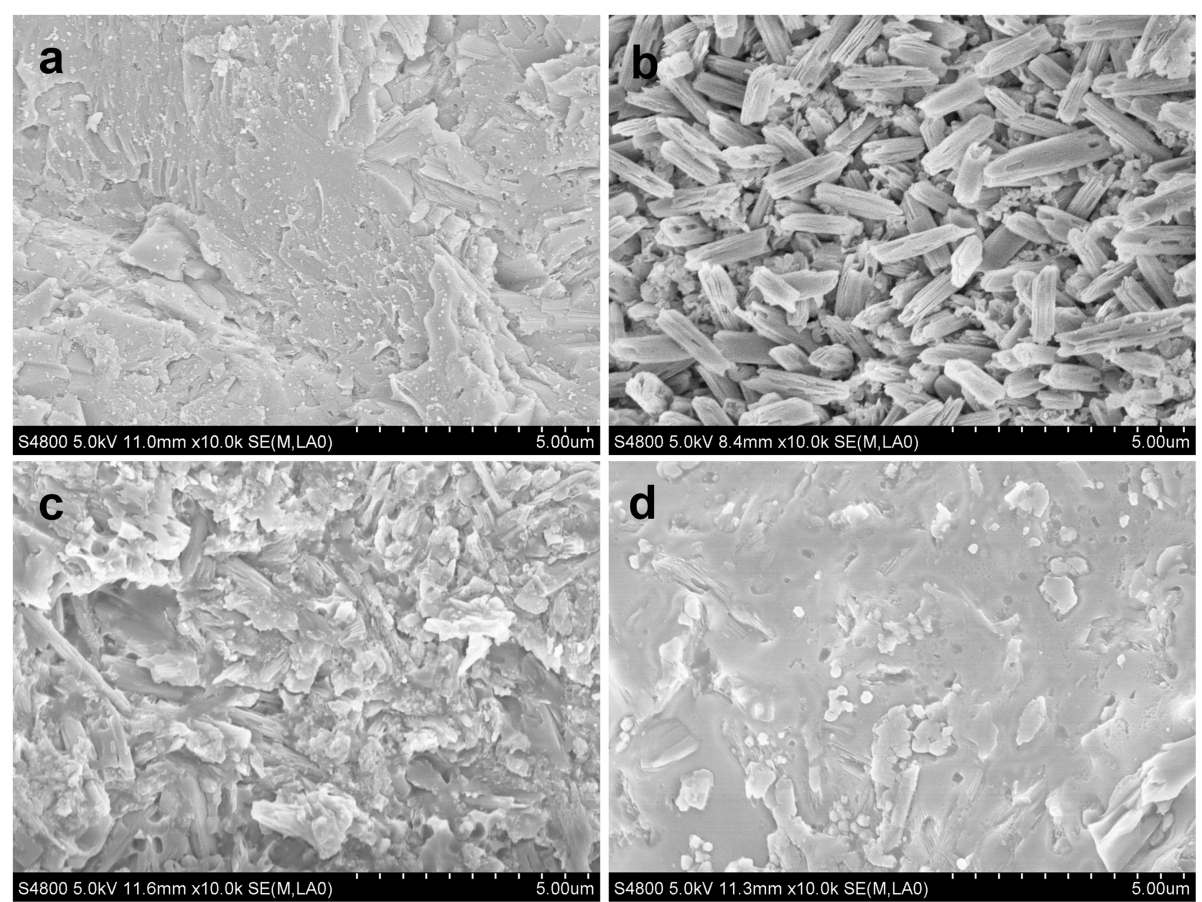

Fig. 7 SEM images of customized ceramic bracket base at $10 \mathrm{kX}$ : a without etching; b hydrofluoric acid etching. And debonding base at $10 \mathrm{kX}$ : $\mathbf{c}$ CCB-E; d CCB-ES

pores were detected (Fig. 7c). However, the relatively smooth and clear surface with silane application was found on CCB-ES base, and some crystal fractures and adhesive fragments were also found (Fig. 7d).

\section{Static and kinetic frictional resistance}

A similar static and kinetic FR values are revealed in Table 2. In comparison, the kinetic groups reached lower values. Except group of 16-SSL, no significant differences $(P \geq 0.05)$ were seen between $\mathrm{CCB}$ and other commercial brackets. The lowest FR value noticed for IC in all subgroups. For identical bracket-ligature combinations with different archwires, FR data indicated that the lower frictional forces with 0.016-in round archwires than those with $0.018 \times 0.025$-in rectangular stainless steel archwires. Although C7 showed close FR values between group 16-SSL and 16-EL, the EL of other brackets except CCB presented a higher FR value than SSL with the same dimensional archwire.

\section{Shear bond strength and adhesive remnant index}

The mean SBS values and ARI scores are shown in Table 3. Multiple comparison test results indicated that IC group (21.02 $\mathrm{MPa}$ ) presented the highest SBS value among all groups. There were no statistically significant differences $(\mathrm{P} \geq 0.05)$ in $\mathrm{SBS}$ values among groups $\mathrm{CCB}$ ES (15.16 MPa), CA (14.02 MPa) and C7 (16.15 MPa). The Tukey's HSD test showed that the SBS values of
Table 2 One-way ANOVA comparing different group static and kinetic frictional resistance in Newton

\begin{tabular}{|c|c|c|c|c|c|c|}
\hline \multirow[t]{2}{*}{ Group } & \multicolumn{3}{|c|}{ Static frictional resistance } & \multicolumn{3}{|c|}{ Kinetic frictional resistance } \\
\hline & Mean & SD & P-value & Mean & SD & P-value \\
\hline $18 \times 25-S S L$ & & & $<0.001$ & & & $<0.001$ \\
\hline$C C B$ & $0.77^{\mathrm{bc}}$ & 0.18 & & $0.70^{\mathrm{bc}}$ & 0.16 & \\
\hline CA & $0.72^{b}$ & 0.20 & & $0.68^{b}$ & 0.18 & \\
\hline C7 & $0.94^{c}$ & 0.15 & & $0.87^{c}$ & 0.14 & \\
\hline IC & $0.52^{\mathrm{a}}$ & 0.08 & & $0.50^{\mathrm{a}}$ & 0.08 & \\
\hline $18 \times 25-E L$ & & & $<0.001$ & & & $<0.001$ \\
\hline $\mathrm{CCB}$ & $0.93^{b}$ & 0.11 & & $0.90^{\mathrm{b}}$ & 0.11 & \\
\hline CA & $1.00^{\mathrm{bc}}$ & 0.19 & & $0.89^{b}$ & 0.14 & \\
\hline C7 & $1.14^{c}$ & 0.17 & & $1.00^{\mathrm{b}}$ & 0.17 & \\
\hline IC & $0.59^{\mathrm{a}}$ & 0.05 & & $0.57^{\mathrm{a}}$ & 0.05 & \\
\hline 16-SSL & & & $<0.001$ & & & $<0.001$ \\
\hline $\mathrm{CCB}$ & $0.75^{\mathrm{c}}$ & 0.17 & & $0.70^{c}$ & 0.14 & \\
\hline CA & $0.55^{\mathrm{b}}$ & 0.13 & & $0.52^{b}$ & 0.13 & \\
\hline C7 & $0.53^{b}$ & 0.15 & & $0.48^{\mathrm{b}}$ & 0.14 & \\
\hline IC & $0.33^{\mathrm{a}}$ & 0.03 & & $0.30^{\mathrm{a}}$ & 0.05 & \\
\hline 16-EL & & & $<0.001$ & & & $<0.01$ \\
\hline $\mathrm{CCB}$ & $0.48^{\mathrm{a}}$ & 0.10 & & $0.44^{\mathrm{a}}$ & 0.08 & \\
\hline$C A$ & $0.65^{b}$ & 0.06 & & $0.54^{b}$ & 0.07 & \\
\hline C7 & $0.54^{\mathrm{a}}$ & 0.05 & & $0.45^{\mathrm{ab}}$ & 0.05 & \\
\hline IC & $0.45^{\mathrm{a}}$ & 0.08 & & $0.44^{\mathrm{a}}$ & 0.07 & \\
\hline
\end{tabular}

Mean values with the same superscript letters in common group were not statistically different $(P \geq 0.05)$ 
Table 3 Shear bond strength values and adhesive remnant index scores of all tests groups

\begin{tabular}{|c|c|c|c|c|c|c|c|c|}
\hline \multirow[t]{2}{*}{ Group } & \multicolumn{3}{|c|}{ Shear bond strength } & \multicolumn{4}{|c|}{ Adhesive remnant index } & \multirow[b]{2}{*}{ Mean } \\
\hline & Mean & SD & P-value & ARI 0 & ARI 1 & ARI 2 & ARI 3 & \\
\hline$\overline{C C B-E}$ & $10.21^{\mathrm{a}}$ & 2.30 & $<0.001$ & - & - & - & 10 & 3 \\
\hline CCB-ES & $15.16^{\mathrm{b}}$ & 2.67 & & - & 1 & 5 & 4 & 2.3 \\
\hline CA & $14.02^{\mathrm{b}}$ & 2.00 & & - & - & 1 & 9 & 2.9 \\
\hline ( & $16.15^{\mathrm{b}}$ & 2.56 & & - & 7 & 2 & 1 & 1.4 \\
\hline IC & $21.02^{c}$ & 2.00 & & 1 & - & 3 & 6 & 2.4 \\
\hline $\mathrm{DQ}$ & $10.09^{a}$ & 1.07 & & - & 4 & 4 & 2 & 1.8 \\
\hline
\end{tabular}

Mean values with the same superscript letters were not statistically different $(P \geq 0.05)$. ARI 0, no composite remained on the tooth surface; ARI 1, less than $50 \%$ of composite remained on the tooth surface; ARI 2, more than $50 \%$ of the composite remained on the tooth; ARI 3, all of the composite remained on the tooth, with distinct impression of the bracket mesh

CCB-ES significantly improved $(P<0.05)$ after etching and silane treatments. The highest ARI score 3 was observed for CCB-E group and all brackets keep no residual cement on the base surface. The ARI data indicated that adhesive failures at the ceramic-resin interface were the predominant mode of failure in debonding groups except $\mathrm{C} 7$ and $\mathrm{DQ}$.

\section{Discussion}

This customized system addressed two problems traditionally associated with labial fixed appliances: first, current metallic customized appliances fail to achieve the esthetic appearance as ceramic brackets do. Second, customized design and manufacture of ceramic brackets are technically limited which adversely affect the development of ceramic brackets. Aiming at these problems, $\mathrm{CCB}$ was designed and fabricated by employing CAD technology, 3D printing technology, heat-pressing technology and multi-color lithium disilicate materials.

Virtual treatment planning provides an effective visualization of the final leveling and alignment of the individual arches, so that orthodontists and patient could visually get to know the treatment process and results. Customized design and fabrication not only allows us to reduce bracket thickness to a minimum, but also matches the teeth surface morphology and appearance (color and transparency) to satisfy the specific clinical needs. Besides, the multi-color and multi-transparency system provides a larger tolerance to CCB to imitate various teeth appearances. It is true that customized system does require more time for bracket design and manufacture, but the form-fit properties between the custom bracket base and tooth could achieve a positive lock that makes a correct positioning [22]. Hence, The CCB system is deemed as a promising strategy to provide higher quality of orthodontic treatment by reducing discomfort, chair time and treatment duration and achieving better treatment results.
The high fracture toughness and flexural strength of lithium disilicate materials facilitate the manufacturing ceramic orthodontic brackets. And benefited from lostwax technique, there are no needs to prefabricate a mold as usual and the duration of fabrication can be reduced significantly. SEM results confirmed that CCB performed a smooth and high integrity surface and slot after the procedures of grinding, polishing and glazing.

In orthodontic sliding mechanics, there are many variables to affect the magnitude of the frictional force [23, 24]. Both static and kinetic friction results indicate that ligature and archwire shape have caused different effects for different brand brackets. From Table 2, two basic principles can be observed. The lager the archwire, the higher FR value; elastic ligature can cause the higher FR values than stainless steel wire except CCB. Higher values could be attributed to the increase of friction factor caused by larger contact areas at the interface of wire-slot and wire-ligature. Meanwhile, it should be noted that different brand of ceramic bracket has a different contour and size. When archwire was ligated in slot, excessive size of bracket and archwire certainly would increase the deformation force of elastic ring and lead to larger vertical pressure for archwire. In comparison, stainless steel wire ligature provides a constant initial force by the same operator.

Slot of CCB was polished with ruby oilstone slice, which tends to cause a sharp corner on slot edges. The excessive force of ligaturing would give rise to some permanent deformations of archwire and form notch, especially for smaller round wires at interface of wire and bracket corner. Tooth movement stops when a notched wire catches on the bracket corner and resumes only when the notch is released [25]. When combined with thinner round wires, the sliding resistance of CCB could attribute to the physical notching and deformation of wire instead of contact friction. Therefore, the FR variation regularity of $\mathrm{CCB}$ was inconsistent with that of other three ceramic brackets. Overall, the statistical analyses of FR results with different archwires and ligature combinations suggested that $\mathrm{CCB}$ has an approximate frictional property with commercial brackets $\mathrm{CA}, \mathrm{C} 7$ and IC.

Because ceramic materials do not bond chemically with adhesives, ceramic brackets derive their bond strength from the use of a silane coupling agent on the base of the bracket, through mechanical retention, or both [26]. Therefore, conventional ceramic brackets bases comprise various designs for increasing the surface area. Alumina abrasion blasting was used to divest the investment materials and create an initial morphology of CCB base (Fig. 7a). Although the abrasive blasting process had formed a rough topography on base surface, it still cannot supply cleaned and enough 
micromechanical bonding (interlocking, retention) surface. After etching, it could be observed that HF acid dissolved glassy contents and exposed the crystal structures (Fig. 7b). Research has shown that silane has the potential to react with hydroxyl groups present on the surface of silica in ceramics and the methacrylate group of bonding agent or resin cement [27]. In this study, microcosmic smoother debonding surface and massive adhesive remnants of CCB-ES (Fig. 7d) supports that stronger chemical bond exist at the interface of ceramic and adhesive, not just some micro-retentions like CCB-E (Fig. 7c).

Some researchers suggested that bond strengths between 6 and $8 \mathrm{MPa}$ are clinically sufficient for bonding of brackets to enamel [28, 29]. And, too high bond strength could result in damage to enamel and existing restorations. Thus, adhesive failures and high ARI score are more favorable to avoid enamel fractures during debonding. In this study, all groups achieved mean values higher than $10 \mathrm{MPa}$, and no damages were observed to the debonded tooth surface. CCB-E and CCBES groups both reached an adequate performance while the bonding and debonding procedures. In addition, CCB base surface allows the treatment of hydrofluoric acid, which gives the ability of clinical re-bonding. Consequently, the CCB system could be considered sufficient and reasonable for clinical applications.

\section{Conclusion}

CCB system makes it possible to realize masscustomization for ceramic orthodontic brackets via personalized design, 3D printing technique, heat-pressing technique, and lithium disilicate materials. Within the limitations of this study, the following conclusions were drawn.

1. CCB attained the multi-color appearance and individual contour by means of lithium disilicate materials and custom molding design.

2. Except 16-SSL group, there were no statistically significant differences between $\mathrm{CCB}$ and certain commercial ceramic brackets $(P \geq 0.05)$.

3. The SBS of all groups exhibited higher values than the minimum orthodontic bracket bond strength range of 6-8 MPa, and no statistically significant differences in SBS were observed among CCB-ES, CA and C7 groups $(P \geq 0.05)$. CCB-E attained the highest mean ARI score 3 , and no enamel fractures were detected.

\section{Abbreviations}

ARI: Adhesive remnant index; C7: Crystalline VII; CA: Clarity ADVANCED; CCB: customized ceramic bracket; DQ: Damon Q; FR: Frictional resistance; IC: Inspire ICE; SBS: Shear bond strength

Acknowledgements

Not applicable.

\section{Authors' contributions}

This study was LY's M.D thesis under the guidance of his mentor, GY. The planning of this study, treatment of patients, data collection, data analyses was carried out by all authors. LY prepared the manuscript for publication. All authors read, discussed and approved the final manuscript.

\section{Funding}

This work was supported by grants from National key R \& D Program Project (2018YFC1105703), National Natural Science Foundation of China (81801019) and Sichuan Science and Technology Program (2019JDRC0099).

\section{Availability of data and materials}

The data supporting the findings of this research can be obtained directly from the authors as well as the College of materials science and engineering, Sichuan University, Chengdu, China.

\section{Ethics approval and consent to participate}

This project was approved by the Ethical Committee of West China Hospital of Stomatology (Protocol number: WCHSIRB-D-2018-070), Chengdu, China. All participants/parents carefully read and signed the consent forms.

\section{Consent for publication}

Since this was a M. D thesis, the participants were informed that their intraoral photographs might be used for presentations and publications. None of the participants/parents objected to the use of their intraoral photographs. All authors read and approved the final manuscript.

\section{Competing interests}

The authors declare that they have no competing interests.

\section{Author details}

${ }^{1}$ College of materials science and engineering, Sichuan University, No.24 South Section 1, Yihuan Road, Chengdu, China. ${ }^{2}$ State Key Laboratory of Oral Diseases, National Clinical Research Centre for Oral Diseases, West China Hospital of Stomatology, Sichuan University, Chengdu, No. 14, South Renmin Road, Chengdu, China. ' ${ }^{3}$ epartment of Orthodontics, Ninth People's Hospital Affiliated to Shanghai Jiao Tong University, School of Medicine, No. 639 Zhizaoju Road, Shanghai, China.

Received: 15 June 2019 Accepted: 11 September 2019

Published online: 14 October 2019

\section{References}

1. van der Zande MM, Gorter RC, Wismeijer D. Dental practitioners and a digital future: an initial exploration of barriers and incentives to adopting digital technologies. Br Dent J. 2013;215:E21.

2. Bauer JC, Brown WT. The digital transformation of oral health care Teledentistry and electronic commerce. J Am Dent Assoc. 2001;132:204-9.

3. De Vos W, Casselman J, Swennen GRJ. Cone-beam computerized tomography $(\mathrm{CBCT})$ imaging of the oral and maxillofacial region: a systematic review of the literature. Int J Oral Max Surg. 2009;38:609-25.

4. Müller-Hartwich R, Jost-Brinkmann P, Schubert K. Precision of implementing virtual setups for orthodontic treatment using CAD/CAM-fabricated custom archwires. J Orofa Orthop. 2016;77:1-8.

5. Walton DK, Fields HW, Johnston WM, Rosenstiel SF, Firestone AR, Christensen JC. Orthodontic appliance preferences of children and adolescents. Am J Orthod Dentofac Orthop. 2010;138:691-8.

6. Fujiyama K, Honjo T, Suzuki M, Matsuoka S, Deguchi T. Analysis of pain level in cases treated with Invisalign aligner: comparison with fixed edgewise appliance therapy. Prog Orthod. 2014;15:1-7.

7. Brown MW, Koroluk L, Ko C, Zhang K, Chen M, Nguyen T. Effectiveness and efficiency of a CAD/CAM orthodontic bracket system. Am J Orthod Dentofac Orthop. 2015;148:1067-74.

8. Wiechmann D. A new bracket system for lingual orthodontic treatment part 2: first clinical experiences and further development. J Orofa Orthop. 2003; 64:372-88.

9. Alford TJ, Roberts WE, Hartsfield JK, Eckert GJ, Snyder RJ. Clinical outcomes for patients finished with the SureSmile ${ }^{\mathrm{TM}}$ method compared with conventional fixed orthodontic therapy. Angle Orthod. 2011;81:383-8. 
10. Weber NDJ, Koroluk LD, Phillips C, Nguyen T, Proffit WR. Clinical effectiveness and efficiency of customized vs. conventional preadjusted bracket systems. J Clin Orthod. 2013;47:261.

11. Stamm T, Hohoff A, Ehmer U. A subjective comparison of two lingual bracket systems. Eur J Orthod. 2005;27:420-6.

12. Slater RD. Speech and discomfort during lingual orthodontic treatment. J Orthod. 2014;40:534-7.

13. Birnie D. Ceramic brackets. Br J Orthod. 1990;17:71-5.

14. Kukiattrakoon B, Samruajbenjakul B. Shear bond strength of ceramic brackets with various base designs bonded to aluminous and fluorapatite ceramics. Eur J Orthod. 2010;32:87-93.

15. Lopes Filho H, Maia LEG, Araújo MVA, Ruellas ACO. Influence of optical properties of esthetic brackets (color, translucence, and fluorescence) on visual perception. Am J Orthod Dentofac Orthop. 2012;141:460-7.

16. Yu B, Lee Y. Aesthetic colour performance of plastic and ceramic brackets an in vitro study. J Orthod. 2011;38:167-74.

17. Lee YK. Colour and translucency of tooth-coloured orthodontic brackets. Eur J Orthod. 2008;30:205-10.

18. Galante R, Figueiredo-Pina CG, Serro AP. Additive manufacturing of ceramics for dental applications: a review. Dent Mater. 2019.

19. Dawood A, Marti BM, Sauret-Jackson V, Darwood A. 3D printing in dentistry Br Dent J. 2015:219:521-9.

20. Motro PFK, Kursoglu P, Kazazoglu E. Effects of different surface treatments on stainability of ceramics. J Prosthet Dent. 2012;108:231-7.

21. Artun J, Bergland S. Clinical trials with crystal growth conditioning as an alternative to acid-etch enamel pretreatment. Am J Orthod. 1984:85:333-40.

22. Wiechmann D, Rummel V, Thalheim A, Simon JS, Wiechmann L. Customized brackets and archwires for lingual orthodontic treatment. Am J Orthod Dentofac Orthop. 2003;124:593-9.

23. Read-Ward GE, Jones SP, Davies EH. A comparison of self-ligating and conventional orthodontic bracket systems. Br J Orthod. 1997;24:309-17.

24. Nishio C, Da Motta AFJ, Elias CN, Mucha JN. In vitro evaluation of frictional forces between archwires and ceramic brackets. Am J Orthod Dentofac Orthop. 2004;125:56-64.

25. Burrow SJ. Friction and resistance to sliding in orthodontics: a critical review. Am J Orthod Dentofac Orthop. 2009;135:442-7.

26. Chen H, Su M, Chang HF, Chen Y, Lan W, Lin C. Effects of different debonding techniques on the debonding forces and failure modes of ceramic brackets in simulated clinical set-ups. Am J Orthod Dentofac Orthop. 2007;132:680-6.

27. Söderholm KJM, Shang SW. Molecular orientation of Silane at the surface of colloidal silica. J Dent Res. 1993;72:1050-4.

28. Retief DH. Failure at the dental adhesive-etched enamel interface. J Oral Rehabil. 1974;1:265-84.

29. Reynolds I. A review of direct orthodontic bonding. Br J Orthod. 1975;2:171-8.

\section{Publisher's Note}

Springer Nature remains neutral with regard to jurisdictional claims in published maps and institutional affiliations.

\section{Submit your manuscript to a SpringerOpen ${ }^{\circ}$ journal and benefit from:}

- Convenient online submission

- Rigorous peer review

- Open access: articles freely available online

- High visibility within the field

- Retaining the copyright to your article

Submit your next manuscript at $\boldsymbol{\nabla}$ springeropen.com 